\title{
SB8: A Bevacizumab Biosimilar
}

\author{
Yahiya Y. Syed $^{1}$ \\ ๑) Springer Nature 2020, corrected publication 2020
}

\begin{abstract}
SB8 is a biosimilar of the monoclonal anti-VEGF antibody bevacizumab and is approved in the EU for use in the same types of cancer as bevacizumab. SB8 has similar physicochemical and pharmacodynamic properties to those of reference bevacizumab and pharmacokinetic equivalence was shown in healthy volunteers and patients with non-small cell lung cancer (NSCLC). SB8 demonstrated equivalent clinical efficacy to reference bevacizumab in patients with metastatic or recurrent nonsquamous NSCLC, with similar tolerability, safety and immunogenicity profiles.
\end{abstract}

\section{Key Points}

Biosimilar to bevacizumab

Equivalent efficacy and similar tolerability to reference bevacizumab in patients with metastatic or recurrent nonsquamous NSCLC

Approved for same types of cancer for which bevacizumab is approved

\section{Introduction}

SB8 is a biosimilar of the monoclonal anti-vascular endothelial growth factor (VEGF) antibody bevacizumab. The product details are summarized in Table 1. SB8 is approved in the EU for same types of cancer as bevacizumab (Table 2)

Enhanced material for this Adis Biosimilar Brief can be found at https://doi.org/10.6084/m9.figshare.13175600.

The manuscript was reviewed by: $\boldsymbol{K}$. Araki, Department of Medical Oncology, Gunma Prefectural Cancer Center, Gunma, Japan; P. Gascon, Laboratory of Molecular and Translational Oncology, CELLEX, Barcelona, Spain, S. Gathers, Community Response, Medical University of South Carolina Charleston, Charleston, SC, USA.

Yahiya Y. Syed

demail@ springer.com

1 Springer Nature, Private Bag 65901, Mairangi Bay, Auckland 0754, New Zealand
[1]. SB8 has similar physicochemical, functional and pharmacodynamic characteristics to those of reference bevacizumab [2,3] and pharmacokinetic similarity of the agents has also been demonstrated [3,4]. This article summarizes, from an EU perspective, the key features of SB8 and its clinical use in the treatment of various cancers, focusing on non-small cell lung cancer (NSCLC).

\section{Clinical Pharmacology}

SB8 has similar physiochemical characteristics and functional properties to those of reference bevacizumab (Table 3) $[2,3]$. In vitro functional assay data are supported by comparable anti-tumour activity of SB8 and reference bevacizumab in a human NSCLC xenograft mice model [2].

Pharmacokinetic similarity of SB8 to EU-sourced bevacizumab was demonstrated in a randomized, double-blind, phase 1 pharmacokinetic study in healthy volunteers [4]. The $90 \%$ CI for geometric least squares means ratios for the primary endpoints of area under the concentration-time curve (AUC) from time zero to infinity, AUC from time zero to the last quantifiable concentration and the maximum observed serum concentrations $\left(\mathrm{C}_{\max }\right)$ were within the prespecified bioequivalence margin of $80.00-125.00 \%$ [4]. Furthermore, in a phase 3 study in patients with NSCLC (Sect. 3), $\mathrm{C}_{\max }$ and trough serum concentrations at cycles 1, 3, 5 and 7 were largely similar for SB8 and EUsourced bevacizumab [3]. SB8 had a slightly lower exposure than reference bevacizumab, which did not impact the efficacy or safety of SB8 [2]. Based on the totality of all pharmacological data, bioequivalence of SB8 to EUsourced reference bevacizumab was demonstrated [2]. 
Finished medicinal product A clear to slightly opalescent, colourless to pale brown, sterile and preservative-free solution. A sterile concentrate for solution for infusion containing $100 \mathrm{mg}$ of bevacizumab in a $4 \mathrm{~mL}$ vial or $400 \mathrm{mg}$ bevacizumab in a $16 \mathrm{~mL}$ vial (strength $25 \mathrm{mg} / \mathrm{mL}$ ) [2]

To ensure that a $4 \mathrm{~mL}$ nominal volume can be withdrawn from the vial, there is an overfill of $\approx 0.6 \mathrm{~mL}$. To ensure that a $16 \mathrm{~mL}$ nominal volume can be withdrawn from the vial, there is an overfill of $\approx 1.2 \mathrm{~mL}$ [7]

\begin{tabular}{ll}
\hline Formulation & Trehalose dihydrate, sodium acetate trihydrate, acetic acid, polysorbate 20 and water for injections [2] \\
\hline Stability & Unopened vial: at $30 \pm 2{ }^{\circ} \mathrm{C}$ for 1 month [5] \\
\hline & Opened vial: at $5 \pm 3{ }^{\circ} \mathrm{C}$ for $72 \mathrm{~h} \mathrm{[5]}$ \\
\hline Opened vial: at $5 \pm 3{ }^{\circ} \mathrm{C}$ for 35 days [7] \\
\hline \\
After dilution $\left(1.4\right.$ or $16.5 \mathrm{mg} / \mathrm{ml}$ in $100 \mathrm{ml} 0.9 \% \mathrm{NaCl}$ polyolefin bags): at $5 \pm 3{ }^{\circ} \mathrm{C}$ for 45 days and then 3 days at \\
$30 \pm 2{ }^{\circ} \mathrm{C} \mathrm{[5]}$
\end{tabular}

Table 2 SB8 prescribing summary in the EU $[1]^{\mathrm{a}}$

Approved indications

Colon or rectal carcinoma Breast cancer

Non-small cell lung cancer

Renal cancer

Epithelial ovarian, fallopian tube or primary peritoneal cancer $^{\mathrm{b}}$

Cervical carcinoma
In combination with fluoropyrimidine-based chemotherapy in adult patients with metastatic disease In combination with paclitaxel as first-line treatment of adult patients with metastatic disease

In combination with capecitabine as first-line treatment of adults with metastatic disease in whom other chemotherapy options including taxanes or anthracyclines are not appropriate, excluding patients who received adjuvant taxanes or anthracycline-containing regimens in the last 12 months

In addition to platinum-based chemotherapy as first-line treatment of adult patients with unresectable advanced, metastatic or recurrent disease other than predominantly squamous cell histology

In combination with erlotinib as first-line treatment of adults with unresectable advanced, metastatic or recurrent non-squamous disease with epidermal growth factor receptor activating mutations

In combination with interferon- $\alpha-2 \mathrm{a}$ as first-line treatment of adults with advanced and/or metastatic disease

In combination with carboplatin and paclitaxel as front-line treatment of adult patients with advanced (International Federation of Gynaecology and Obstetrics stages III B, III C and IV) disease

In combination with carboplatin and gemcitabine or carboplatin and paclitaxel in adult patients with first recurrence of platinum-sensitive disease who have not received prior therapy with bevacizumab or other VEGF inhibitors or VEGF receptor targeted agents

In combination with topotecan or pegylated liposomal doxorubicin in adult patients with platinum-resistant recurrent disease who received no more than two prior chemotherapy regimens and who have not received prior therapy with bevacizumab or other VEGF inhibitors or VEGF receptor targeted agents

In combination with paclitaxel and cisplatin (or paclitaxel and topotecan in patients who cannot receive platinum therapy) in adult patients with persistent, recurrent, or metastatic disease

${ }^{a}$ Consult local prescribing information for details including pre- and post-medications, contraindications, warning and precautions

${ }^{\mathrm{b}} \mathrm{SB} 8$ is not indicated for use in combination with paclitaxel for the treatment of adult patients with platinum resistant recurrent disease

SB8 was stable under extreme aseptic conditions [5]. Unopened SB8 vials and in-use SB8 (opened vials and diluted product) at room temperature or refrigerated conditions did not show clinically relevant changes in physicochemical stability and functional characteristics and potential safety-related properties (Table 1) [5].

\section{Clinical Efficacy}

SB8 showed equivalent efficacy to reference bevacizumab in a randomized, double-blind, multicentre phase 3 study in patients with metastatic or recurrent nonsquamous
NSCLC (reference indication) [3]. Eligible patients were adults (age $\geq 18$ years) with histologically and/or cytologically confirmed disease who had $\geq 1$ measurable lesion (defined by RECIST version 1.1), Eastern Cooperative Oncology Group (ECOG) performance status of 0 or 1 and a life expectancy of $\geq 3$ months. Patients who received systemic treatment for metastatic or recurrent NSCLC, systemic neoadjuvant or adjuvant chemotherapy within 12 months before randomization, or VEGF- or epidermal growth factor receptor-targeting agents were among those excluded. Stratified by age $(<70$ and $\geq 70$ years) and gender, patients received intravenous SB8 $(n=379)$ 
A recombinant humanised monoclonal antibody produced in Chinese Hamster Ovary cells that selectively binds to human VEGF, thereby inhibits the binding of VEGF to its receptors, Flt-1 and KDR on endothelial cell surface; this action reduces tumour vascularization, resulting in tumour growth inhibition [2]

Physicochemical characterization Similar to EU-sourced reference bevacizumab with respect to primary and higher order structure, charged variants, and purities and impurities, with minor differences not considered clinically relevant [2]

Pharmacodynamic similarity

Similar to EU-sourced reference bevacizumab with respect to binding affinity to VEGF-A, FcRn and C1q, and potency for VEGF neutralization, VEGF phosphorylation inhibition, and HUVEC anti-proliferation, -migration, and -survival activity [2]

Pharmacokinetic similarity

Similarity between SB8 and EU-sourced reference bevacizumab was demonstrated as geometric LSM ratios and their $90 \%$ CIs for $\mathrm{AUC}_{\infty}, \mathrm{AUC}_{\text {last }}$ and $\mathrm{C}_{\max }$ (primary endpoints) were within the prespecified acceptance range of $0.80-1.25$ in healthy volunteers [4]

$\mathrm{C}_{\text {trough }}$ and $\mathrm{C}_{\max }$ values at cycles 1, 3, 5 and 7 for SB8 and EU-sourced reference bevacizumab were largely similar in patients with NSCLC [3]

Immunogenicity

Comparable between SB8 and EU-sourced reference bevacizumab; the potential impact of ADAs on pharmacokinetics, efficacy and safety of SB8 was not considered clinically relevant [2]

Efficacy and tolerability

Equivalent efficacy and similar tolerability and safety to reference bevacizumab in patients with metastatic or recurrent nonsquamous NSCLC [3]

$A D A$ anti-drug antibodies, $A U C$ area under the concentration-time curve, $A U C_{\infty}$ AUC from time zero to infinity, $A U C_{\text {last }}$ AUC from time zero to time of last measurable concentration, $C_{\max }$ maximum serum concentration, $C_{\text {trough }}$ trough serum concentration, $H U V E C$ human umbilical vein endothelial cells, $L S M$ least squares mean, $N S C L C$ non-small cell lung cancer, $V E G F$ vascular endothelial growth factor

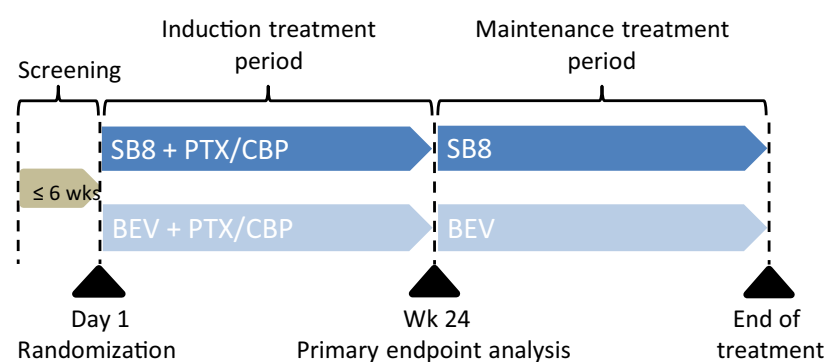

Fig. 1 Design of the SB8-G31-NSCLC trial [3]. BEV reference bevacizumab, $C B P$ carboplatin, $P T X$ paclitaxel, $w k(s)$ week(s)

or reference bevacizumab $(n=384) 15 \mathrm{mg} / \mathrm{kg}$, coadministered with paclitaxel and carboplatin, every 3 weeks for up to six cycles (induction treatment). Patients with complete response (CR), partial response (PR) or stable disease at the end of induction treatment received maintenance therapy with SB8 or reference bevacizumab every 3 weeks until disease progression or unacceptable toxicity (Fig. 1) [3].

The primary endpoint was best overall response rate [ORR] (CR + PR, based on RECIST version 1.1) during the induction treatment period by 24 weeks [3]. Equivalence was established if the 2-sided $90 \%$ CI for the best ORR risk ratio was within $0.737-1.357$ in the full analysis set (FAS) or if the 2-sided $95 \% \mathrm{CI}$ for the best ORR risk difference was within $-12.5 \%$ to $12.5 \%$ in the per-protocol set (PPS) [3].

Baseline demographics and disease characteristics were similar in SB8 and reference bevacizumab groups [3]. Mean age was 60.1 years and $66.6 \%$ of patients were men; the majority were White $(91.1 \%)$, had an ECOG performance status of $1(72.0 \%)$ and had stage IV disease $(99.0 \%)$. The median duration of disease was 1.1 months [3].

SB8 was equivalent to reference bevacizumab [3]. In the FAS, the best ORR was 47.6 and $42.8 \%$, respectively; the best ORR risk ratio $(1.11 ; 90 \%$ CI $0.975-1.269)$ was within the prespecified equivalence margin. In the PPS, the best ORR was $50.1 \%$ and $44.8 \%$, respectively; the best ORR risk difference was $5.3 \%$ (95\% CI $-2.2 \%$ to $12.9 \%$ ) and thus, only the lower margin, but not the upper margin, was within the prespecified equivalence margin. The robustness of the primary analysis was supported by sensitivity analyses, using age groups ( $<70, \geq 70$ years), gender (male, female), geographic region (EU, non-EU) and treatment groups as covariates [3].

In the FAS, at the end of study, SB8 was also comparable to reference bevacizumab with respect to median progression-free survival [PFS] (8.50 vs 7.90 months; HR 0.99; 95\% CI, 0.83-1.18), median overall survival [OS] (14.90 vs 15.80 months; HR 1.03; 95\% CI, 0.83-1.28), median duration of response (7.70 vs 7.00 months; HR 1.05; 95\% CI 0.81-1.37), 12 -month PFS rate (34 vs 30\%) and 12-month OS rate (61 vs $62 \%$ ) [3].

In exploratory analyses, 95\% CIs for the best ORR risk difference between the SB8 and reference bevacizumab groups by weeks 11 and 17 of the induction treatment was within the prespecified equivalence margin in the PPS [6]. Similar results were seen by week 24 in subgroups based on age, gender, race, geographical region, ECOG performance status, smoking status, cancer type and distant metastases [6]. SB8 was also comparable to reference bevacizumab with respect to the mean maximum percentage change from baseline in tumour burden by weeks 11,17 and 24 of the induction treatment period $[3,6]$. 


\section{Tolerability and Safety}

The tolerability and safety profile of SB8 was generally similar to that of reference bevacizumab in the phase 3 trial [3]. The mean number of treatment cycles was similar in SB8 and reference bevacizumab groups during induction period (4.8 vs 4.8 ) and maintenance period (9.3 vs 9.1). In the safety set ( $n=378$ and 380 in SB8 and reference bevacizumab groups), treatment-emergent adverse events (TEAEs) occurred in the majority of patients $(92.1 \mathrm{vs} 91.1 \%)$, with grade $\geq 3$ TEAEs reported in 46.0 and $40.8 \%$ of patients. The most common (incidence $\geq 20 \%$ ) TEAEs of any grade were alopecia (48.7 vs $48.2 \%$ ), anaemia ( 24.3 vs $23.7 \%$ ) and nausea (19.6 vs $21.1 \%$ ); the most common grade $\geq 3$ TEAEs were neutropenia (8.7 vs $9.5 \%$ ), hypertension (6.3 vs $3.7 \%$ ), anaemia ( 4.8 vs $5.5 \%$ ) and decreased neutrophil count (4.0 vs $3.2 \%$ ). TEAEs of special interest were grade $\geq 3$ hypertension ( 7.7 vs $4.2 \%$ ) and grade $\geq 2+$ proteinuria on urine dipstick/urinalysis ( $0.5 \%$ vs $1.8 \%)$; other important TEAEs with an incidence $\geq 2 \%$ included hypersensitivity reactions/infusion-related reactions (12.7 vs. $12.4 \%)$, bleeding/haemorrhage (11.4 vs. $11.8 \%$ ), pulmonary haemorrhage (3.4 vs $4.5 \%$ ), venous thromboembolic events (3.2 vs. $3.7 \%$ ), and congestive heart failure (2.1 vs. $2.4 \%$ ). Serious TEAEs occurred in 19.8 and $21.3 \%$ of SB8 and reference bevacizumab recipients, with 13.2 and $9.5 \%$ of patients discontinuing study medication because of TEAEs. The incidence of death (regardless of causality) was 5.8 and $7.1 \%$ [3].

\section{Immunogenicity}

The immunogenicity of SB8 was similar to that of reference bevacizumab, with no new safety signals attributable to the immunogenicity of SB8 $[2,3]$. In the phase 3 trial, antidrug antibodies (ADAs) were detected in 16.1 and $11.0 \%$ of patients in the SB8 and reference bevacizumab groups up to the end of treatment [3]. Neutralizing antibodies were detected in 7.1 and $8.3 \%$ of patients at the end of treatment [2]. Best ORRs tend to be higher with SB8 versus reference bevacizumab in ADApositive patients, while the opposite was true for ADA-negative patients. ADAs had no clinically relevant impact on PFS and duration of response in both groups. In the SB8 group, two cases of treatment discontinuations (anaphylaxis and hypersensitivity) appeared to be related to immunogenicity [2].

\section{Conclusion}

SB8 is a bevacizumab biosimilar with similar efficacy, tolerability, safety, physiochemical characteristics and functional properties to the reference product (Table 3). Based on physicochemical and functional analyses, as well as clinical data in patients with metastatic or recurrent nonsquamous NSCLC (Table 3), SB8 is approved in the EU for the same types of cancer for which bevacizumab is approved (Table 2).

\section{Declarations}

Funding The preparation of this review was not supported by any external funding.

Authorship and Conflict of interest Yahiya Syed is a salaried employee of Adis International Ltd/Springer Nature, and declares no relevant conflicts of interest. All authors contributed to the review and are responsible for the article content.

Ethics approval, Consent to participate, Consent to publish, Availability of data and material, Code availability Not applicable.

Open Access This article is licensed under a Creative Commons Attribution-NonCommercial 4.0 International License, which permits any non-commercial use, sharing, adaptation, distribution and reproduction in any medium or format, as long as you give appropriate credit to the original author(s) and the source, provide a link to the Creative Commons licence, and indicate if changes were made. The images or other third party material in this article are included in the article's Creative Commons licence, unless indicated otherwise in a credit line to the material. If material is not included in the article's Creative Commons licence and your intended use is not permitted by statutory regulation or exceeds the permitted use, you will need to obtain permission directly from the copyright holder. To view a copy of this licence, visit http://creativecommons.org/licenses/by-nc/4.0/.

\section{References}

1. European Medicines Agency. Aybintio $25 \mathrm{mg} / \mathrm{ml}$ concentrate for solution for infusion: summary of product characteristics. https:// www.ema.europa.eu/ (2020). Accessed 4 Sep 2020.

2. European Medicines Agency. Aybintio: assessment report. https:// www.ema.europa.eu/ (2020). Accessed 4 Sep 2020.

3. Reck M, Luft A, Bondarenko I, et al. A phase III, randomized, double-blind, multicenter study to compare the efficacy, safety, pharmacokinetics, and immunogenicity between SB8 (proposed bevacizumab biosimilar) and reference bevacizumab in patients with metastatic or recurrent nonsquamous non-small cell lung cancer. Lung Cancer. 2020;146:12-8.

4. Shin D, Lee YJ, Choi J, et al. A phase I, randomized, single-dose pharmacokinetic study comparing sb8 (bevacizumab biosimilar) with reference bevacizumab in healthy volunteers. Cancer Chemother Pharmacol. 2020;86(4):567-75.

5. Park D, Kim J, Yun J, et al. Evaluation of the physico-chemical and biological stability of SB8 (Aybintio), a proposed biosimilar to bevacizumab, under ambient and in-use conditions. Adv Ther. 2020. https://doi.org/10.1007/s12325-020-01465-0.

6. Planchard D, Shin D, Choi J, et al. Exploratory analyses of efficacy from a phase III study comparing SB8, a proposed bevacizumab biosimilar, and reference bevacizumab in patients with metastatic or recurrent non-squamous (NSCLC) [abstract no. 1377P]. Ann Oncol. 2020;31(Suppl. 4):S877-78.

7. Data on file, Samsung Bioepis (2020). 